\title{
The Landauer Resistance and Band Spectra For the Counting Quantum Turing Machine
}

\author{
Paul Benioff \\ Physics Division, Argonne National Laboratory Argonne, IL 60439, e-mail: \\ pbenioff@anl.gov
}

\begin{abstract}
In other work, the generalized counting quantum Turing machine (GCQTM) was studied. For any $N$ this machine enumerates the first $2^{N}$ integers in succession as binary strings. The generalization consists of associating a potential with read 1 steps only. The Landauer Resistance (LR) and band spectra were determined for the tight binding Hamiltonians associated with the GCQTM for energies below the potential height. Here these calculations are extended to energies both above and below the barrier height. For parameters and potentials in the electron region, The LR fluctuates rapidly between very high and very low values as a function of momentum. The rapidity and extent of the fluctuations increases rapidly with increasing $N$. For $N=18$, the largest value considered, the LR shows good transmission probability as a function of momentum with numerous holes of very high LR values present. This is true for energies both above and below the potential height. It is suggested that the main features of the LR can be explained by coherent superposition of the component waves reflected from or transmitted through or across the $2^{N-1}$ potentials present in the distribution. If this explanation is correct, it provides a dramatic illustration of the effects of quantum nonlocality.
\end{abstract}

\section{Introduction}

Since the early 1980s quantum computation has been under active development in several directions. This includes the development of efficient algorithms [1-3], simulation of physical systems[4,5], quantum error correction [6], and use of quantum gates [7]. In this work and in earlier work on Quantum Turing Machines [8,9], computations were assumed to proceed smoothly with no potentials present to cause reflections or decay of the transmitted state.

The importance of including these potentials and the resulting degradation of performance has been emphasized by Landauer [10]. He has noted that 
potentials can be present because of environmental influences and physical limitations and errors $[11,12]$ which can occur in realistic models.

In previous work $[13,14]$ on quantum Turing machines (QTMs) potentials were introduced by associating them with different types of steps. Isolation from the environment is assumed. In this case the Feynman [15] Hamiltonian for these generalized quantum Turing machines (GQTM)s, can be also be described as a sum of tight binding Hamiltonians each of which has a different potential distribution along a one dimensional path of computation states. Which of these Hamiltonians applies to the GQTM is determined by the initial state.

The effect of these potentials was studied in detail for the counting generalized QTM (GQTM), which enumerates the nonnegative integers in succession as binary strings. The potential distribution associated with this GQTM, is closely related to the heirarchical sequence $[13,16,17]$. This sequence gives the number of $1 s$ appearing before the first 0 in the successive iteration of integers represented as binary strings.

Both the band spectra and the Landauer Resistance $[18,19]$ were calculated as a function of energy for computations which enumerate the first $2^{N}$ integers as binary strings. The calculations were limited to energies below the height of the potential (bound state energies).

In this paper the calculations made for the band spectra and LR for the counting GQTM will be extended by removal of the limitation of the energy range to energies below the barrier. Since the physical model used corresponds to head motion on a 1-D lattice of qubits with a Hamiltonian constructed from step operators, the full energy range remains finite.

In the next section the physical model and main points of the description of the Hamiltonians for GQTMs will be reviewed. The use of the transfer matrix to calculate both the band spectra and LR will be briefly reviewed in Section 3. Following this the counting GQTM will be briefly described. Since the material has already been presented elsewhere, the discussion will be quite brief with additional material incorporated by reference.

Results of calculations of the band spectra and the LR will be presented and discussed in Section 5. The LR, plotted as the $\log L R$ as a function of momentum, will be seen to fluctuate rapidly between very large and very small values. The fluctuations get more rapid and extreme as $N$ is increased and the LR increases as the potential height is increased. The association between the LR and band spectra for the associated periodic system obtained here is similar to that obtained by others $[20,21]$ in that peaks are associated with gaps

In Section 6 problems with extending the calculations to moderate values of 
$N$ are briefly discussed. The possible importance of coherent superposition of wave components reflected from the many potentials in the distribution in producing the observed fluctuations in the LR will be discussed. It is noted that this provides a good example of quantum nonlocality.

\section{Hamiltonians for GQTMs}

\subsection{The Physical Model}

The physical model for QTMs consists of a two-way infinite lattice of systems each of which can assume any state in a finite dimensional Hilbert space $\mathcal{H}_{s}$. If $\mathcal{H}_{s}$ is two dimensional the systems are called qubits, However this term is used here irrespective of the dimensionality of $\mathcal{H}_{s}$. A head, described by a finite basis of states $|l\rangle$ with $l \epsilon L$, moves along the lattice interacting with the lattice systems at or adjacent to the head position. Possible elementary actions include any combination of head motion one site in either direction, lattice system state change, and head state change.

The system states are all assumed to lie in a separable Hilbert space $\mathcal{H}$. A particular basis, the computation basis, defined by the set of states $\{|l, j, S\rangle\}$ and which spans $\mathcal{H}$, is used. Here $l, j$ refer to the internal state and lattice position of the head. The qubit lattice computation basis state $|S\rangle=\otimes_{m=-\infty}^{\infty}|S(m)\rangle$ where $S$ is a function from the integers to the qubit state labels (e.g. $\{0,1\}$ ) such that $S(m) \neq 0$ for at most a finite number of values of $m$. This condition, the 0 tail state condition, is one of many that can be imposed to keep the basis denumerable.

\subsection{Tight Binding Hamiltonians for GQTMs}

A bounded linear operator $T$ is associated with each GQTM. $T$ or its adjoint is referred to as a step operator for the GQTM because iteration of $T$ (or its adjoint) corresponds to the successive steps in the forward (or backward) time direction of the GQTM. An infinitesimal time interval is associated with the steps of $T$ so that it can be used directly to construct a time independent Hamiltonian according to Feynman's prescription [15]:

$$
H=K\left(2-T-T^{\dagger}\right)
$$

where $K$ is a constant. Note that if $T=Y$ where $Y$ is the bilateral shift along the lattice, then $H$ is the kinetic energy of free head motion on the lattice. 
As such it is equivalent to the symmetrized discrete version of the second derivative, $\left(-\hbar^{2} / 2 m\right) d^{2} / d x^{2}$.

For each GQTM the associated step operator $T$ is defined as a finite sum over elementary step operators. That is $T=\sum_{l, s} T_{l s}$ where the sum is over all head state labels $l \epsilon L$ and all qubit state labels $s \epsilon \mathcal{S}$ where $\mathcal{S}$ is the set of qubit state labels. For binary qubits $\mathcal{S}=\{1, \infty\}$. $T_{l, s}$ corresponds to the action taken by the GQTM associated with $T$ when the head in state $|l\rangle$ sees or reads a qubit in state $|s\rangle$.

For each $l, s T_{l, s}=\gamma_{l, s} W_{l, s}$ where $\gamma_{l, s}$ is a positive real constant $\leq 1$. $W_{l, s}$ is a sum over all lattice positions of products of projection operators, bit and head state change, and head position change operators. The specific definition of $W_{l, s}$ appears elsewhere [13].

The generalization from QTMs to GQTMs consists in allowing values of $\gamma_{l, s} \neq 1$. Thus every QTM is a GQTM with $\gamma_{l, s}=1$ for all $l, s$. Note that the generalization does not expand or change the definition of quantum computability.

The main condition imposed on $T$ and its adjoint is that they be distinct path generating in some basis $B$ which spans $\mathcal{H}$. This means that iterations of $T$ or $T^{\dagger}$ on any basis state in $B$, generate a path of states that, up to normalization, are also states in $B$. The paths are distinct in that no two paths join, intersect, or branch.

Although $B$ can be any basis which spans $\mathcal{H}$, (see [22] for details), unless explicitly specified otherwise, $B$ is limited here to be the computation basis, $\{|l, j, S\rangle\}$. A path in the $B$ is defined as a set of states in $B$ ordered by iteration of $T$ or $T^{\dagger}$. If the state $\left|l_{m}, j_{m}, S_{m}\right\rangle=|m, i\rangle$ is the $m$ th state in some path $i$, then the $m+1 s t$ and $m-1 t h$ states in the path are given respectively by $|m+1, i\rangle=W_{l_{m}, S_{m}\left(j_{m}\right)}\left|l_{m}, j_{m}, S_{m}\right\rangle=\left|l_{m+1}, j_{m+1}, S_{m+1}\right\rangle$ and $|m-1, i\rangle=W_{l_{m-1}, S_{m-1}\left(j_{m-1}\right)}^{\dagger}\left|l_{m}, j_{m}, S_{m}\right\rangle=\left|l_{m-1}, j_{m-1}, S_{m-1}\right\rangle$. These equations must be modified if either $T$ or $T^{\dagger}$ annihilate $|m, i\rangle$.

If $T$ is distinct path generating in $B$ then $T$ can be decomposed into a direct sum $T=\sum_{i} T_{i} P_{i}$ where $P_{i}$ projects out a subspace $\mathcal{H}_{i}$ of $\mathcal{H}$ spanned by a subset $B_{i}$ of $B$. For each $i$ iteration of $T$ and $T^{\dagger}$ on the states in $B_{i}$ orders them into a path, denote the computation path $i$. The action of $T_{i}$ and $T_{i}^{\dagger}$ on each state $|m, i\rangle=\left|l_{m}, j_{m}, S_{m}\left(j_{m}\right)\right\rangle$ which is not a terminal path state is given by

$$
\begin{aligned}
T\left|l_{m}, j_{m}, S_{m}\right\rangle & =\gamma_{l_{m}, S_{m}\left(j_{m}\right)}\left|l_{m+1}, j_{m+1}, S_{m+1}\right\rangle \\
T^{\dagger}\left|l_{m}, j_{m}, S_{m}\right\rangle & =\gamma_{l_{m-1}, j_{m-1}, S_{m-1}\left(j_{m-1}\right)}\left|l_{m-1}, j_{m-1}, S_{m-1}\right\rangle
\end{aligned}
$$


Additional details on the decomposition and its description as a sum of weighted shifts is given elsewhere [14].

Corresponding to the decomposition of $T$ is a decomposition of the Hamiltonian, Eq. 1, $H=\sum_{i} H_{i} P_{i}$ where for each $i$,

$$
H_{i}=K\left(2-T_{i}-T_{i}^{\dagger}\right)
$$

As is noted elsewhere [13] $H_{i}$ can also be written in the form

$$
H_{i}=K\left(2-U_{i}-U_{i}^{\dagger}\right)+V_{i}
$$

where $U_{i}$ is a shift along the path $i . U_{i}$ and $U_{i}^{\dagger}$ are defined by Eqs. 2 with $\gamma_{l, s}=1$ for all $l, s$.

$K\left(2-U_{i}-U_{i}^{\dagger}\right)$ is, formally, the kinetic energy associated with the evolution of the computation along path $i$. For the physical model used here it is also the kinetic energy of head motion on the qubit lattice. (The kinetic energy depends on the magnitude but not the direction of the head momentum on the lattice.)

The potential $V_{i}=K\left(U_{i}-T_{i}+U_{i}^{\dagger}-T_{i}^{\dagger}\right)$ is a nearest neighbor off-diagonal potential with matrix elements on internal path states given by

$$
\left\langle m^{\prime}, i\left|V_{i}\right| m, i\right\rangle=K\left[\left(1-\gamma_{l_{m}, S_{m}\left(j_{m}\right)}\right) \delta_{m^{\prime}, m+1}+\left(1-\gamma_{l_{m-1}, S_{m-1}\left(j_{m-1}\right)} \delta_{m^{\prime}, m-1}\right] .(5)\right.
$$

In Schrödinger evolution under the action of $H$, Eq. 1, the choice of which $H_{i}$ is active is determined by the initial state $\Psi(0)$. If $\Psi(0)$ is a superposition of of computation basis states in one path $i$, then $H_{i}$ is the only active component. If $\Psi(0)$ is a superposition of computation basis states in different paths, then all $H_{i}$ are active for just those paths $i$ in which $\Psi(0)$ has a nonzero component. In this case the states in the different paths evolve coherently which corresponds to quantum parallel computation [8].

\section{Transfer Matrix}

For each computation path $i$ one is interested in the evolution of the system state along the path under the action of $H_{i}$. For paths in which the region of nonzero potentials is finite in extent with potential free regions on both sides, the probability of completion of the computation is given by the transmission probability of the state through the potential region. This can be obtained by 
use of the transfer matrix following methods developed by Erdos and Herndon $[19]$.

To be specific, assume the potential is nonzero in a region extending from state $|a, i\rangle$ to $|a+m, i\rangle$ and is zero elsewhere on an infinite path $i$. The states on either side of the region can be written in the form,

$$
\begin{aligned}
\Psi_{I} & =\sum_{j=-\infty}^{a-1}\left(A \mathrm{e}^{\mathrm{ikj}}+\mathrm{Be}^{-\mathrm{ikj}}\right)|\mathrm{j}\rangle \\
\Psi_{I I I} & =\sum_{j=a+m+1}^{\infty}\left(F \mathrm{e}^{\mathrm{ikj}}+\mathrm{Ge}^{-\mathrm{ikj}}\right)|\mathrm{j}\rangle
\end{aligned}
$$

Here $j$ is the path state label (the path label $i$ in $|j, i\rangle$ is suppressed) and $k$ is the momentum in the potential free regions.

The form of $\Psi_{I I}$, the state in the region II of nonzero potential, depends on the form of the potential. In general $\Psi_{I I}=\sum_{j=a}^{a+m}\left(C_{j} \mathrm{e}^{\mathrm{i} \mathrm{l}_{\mathrm{j}} \mathrm{j}}+\mathrm{D}_{\mathrm{j}} \mathrm{e}^{-\mathrm{i} \mathrm{l}_{\mathrm{j}} \mathrm{j}}\right)|\mathrm{j}\rangle$ where the momenta $l_{j}$ and coefficients $C_{j}, D_{j}$ depend on the potential height, Eq, 5 , at $|j\rangle$. Both the momenta and coefficients are constant over subregions of constant potential height. For any subregion for which the energy corresponding to the momentum $l_{j}$ is less than the potential height for the subregion, $l_{j}$ is replaced by $\mathrm{il}_{\mathrm{j}}$. Additional details are given elsewhere $[19,14]$.

The four complex coefficients $A, B, F, G$ are completely determined by boundary conditions for the case under study and the properties of the transfer matrix. For computations it is convenient to let Regions I and III be the initial and final state regions. In this case, the $A, B, F$ coefficient terms of Eq. 6 are respectively the initial incoming state, the reflected term and the transmitted term. In this case $G=0$ as there is no incoming term in the final state region. Different boudary conditions are used if $\Psi$ represents an eigenstate of $H_{i}$.

The transfer matrix $Z$ relates the coefficients $F, G$ to $A, B$ according to [19]

$$
\left.\left.\mid \begin{array}{l}
F \\
G
\end{array}\right) \cdot=Z \mid \begin{array}{l}
A \\
B
\end{array}\right) .
$$

$Z$ is a unimodular (determinant=1) $2 \times 2$ matrix which also satisfies [19]

$$
\begin{aligned}
& Z_{11}=Z_{22}^{*} \\
& Z_{12}=Z_{21}^{*}
\end{aligned}
$$

The above definitions can be iterated to define $Z$ as a product of transfer 
matrices for individual subregions of constant potential. If there are $M$ such regions then $Z$ is the product of $M 2 \times 2$ matrices, taken in the order in which the subregions occur in region II [19].

The Landauer Resistance (LR) [18,19], which measures the resistance to transmission through the potential region, is defined by $L R=|B|^{2} /|F|^{2}$ with $G=0$. For a computation on a path $i$, the probability of completion is given by $1 /(1+L R)=|F|^{2}$. The properties of $Z$ can be used to obtain [19]

$$
L R=\left.|Z|_{12}\right|^{2}
$$

It is clear from the above that the LR depends both on the path and on the system energy (i.e. the energy of the head as it moves on the lattice).

The transfer matrix is also used to determine for each path $i$ the energy band spectra for the infinite 1-D crystal whose unit cell is given by the potential distribution of $H_{i}$, Eq. 5 (the associated periodic system). As is well known $[23,20,21]$ the band and gap distribution is obtained from a plot of $|\operatorname{Tr} Z| / 2$ as a function of momentum or energy. Regions in which $|\operatorname{Tr} Z| / 2 \leq 1$ correspond to bands; regions with $|\operatorname{Tr} Z| / 2>1$ correspond to gaps.

\section{The Counting GQTM}

An interesting example which can be used to illustrate the foregoing is the counting QTM $[13,14]$. Starting with a string of $0 s$ this machine generates nonnegative integers as binary strings in succession by repetitve addition of $1 \bmod 2$. One marker locates the start of the strings on the qubit lattice and another marker, if present, locates the maximum length of the strings.

A step operator $T$ which carries this out can be given as a sum of 7 terms. A simple but nontrivial representation as a generalized QTM is obtained by setting, for all $l, \gamma_{l, s}=1$ for all $s \neq 1$ and $\gamma_{l, 1}=\gamma<1$. The resulting step operator is given by

$$
\begin{aligned}
T= & \sum_{j=-\infty}^{\infty}\left(Q_{0} P_{0 j} u P_{j}+w Q_{0} P_{2 j} u P_{j}+Q_{1} P_{0 j} u P_{j}\right. \\
& +w Q_{1} P_{2 j} u^{\dagger} P_{j}+\gamma Q_{2} v_{x j} P_{1 j} u^{\dagger} P_{j}+w^{\dagger} Q_{2} v_{x j} P_{0 j} u P_{j} \\
& \left.+w Q_{2} P_{2 j} u P_{j}\right)
\end{aligned}
$$

The projection operators $Q_{l}, P_{s, j} P_{j}$ refer to the head in state $|l\rangle$, the site $j$ qubit in state $|s\rangle$, and the head at site $j . w$ is a shift mod 3 on the three head states $\left(w Q_{m}=Q_{m+1} w \bmod 3\right)$ and $u$ shifts the head along the lattice by one 
site $\left(u P_{j}=P_{j+1} u\right)$. The need for markers is accounted for here by choosing the qubits in the lattice to be ternary with states $|0\rangle,|1\rangle,|2\rangle .|2\rangle$ is used as a marker and $|0\rangle,|1\rangle$ are used for binary strings. The qubit transformation operator $v_{x j}=\sigma_{x j}\left(P_{0 j}+P_{1 j}\right)+P_{2 j}$ exchanges the states $|0\rangle,|1\rangle$ and does nothing to the state $|2\rangle$ for the site $j$ qubit.

The adjoint $T^{\dagger}$ is defined from $T$ in the usual way noting that the operators for the head states, head position states, and qubit states commute with one another. Note that $v_{x j}^{\dagger}=v_{x j}$. The explicit form of $T^{\dagger}$ as well as other aspects of this GQTM are given elsewhere [14].

For this GQTM motion from state $|m, i\rangle$ to state $\left|m^{\prime}, i\right\rangle$ is potential free unless the 5 th terms of $T$ or $T^{\dagger}$ are active. In this case, for $|m, i\rangle=\left|l_{m}, j_{m}, S_{m}\left(j_{m}\right)\right\rangle$ $l_{m}=2$ and $S_{m}\left(j_{m}\right)=1$ or(and) $l_{m-1}=2$ and $S_{m-1}\left(j_{m-1}\right)=1$. If either one but not both of these conditions hold, then by Eq, $5, V=K(1-\gamma)$. If both conditions hold then $V=2 K(1-\gamma)$. Equivalent conditions can be given for the state $\left|m^{\prime}, i\right\rangle$.

An analysis of the types of potential barriers that can occur in a distribution for this shows that they are at least 2 path states wide. A barrier that is $m+1$ sites wide, corresponding to $m$ successive (read 1) steps in which the 5 th term of $T$ or $T^{\dagger}$ is active, has a central core of height $V=2 K(1-\gamma)$ occupying $m-1$ successive path sites or states. The core is flanked by two potentials of height $V=K(1-\gamma)$ occupying just one site or state. For $m=1$ the barrier occupies 2 sites and is of height $K(1-\gamma)$.

For potential free regions the relation between energy and momentum is given by $E=2 K(1-\cos k)$. For the core region of a barrier $E=2 k(1-\gamma \cos l$ ) (or $E=2 K(1-\gamma \cosh l)$ if $E<V)$. From these equations one obtains $\cos k=$ $\gamma \cos l($ or $\cos k=\gamma \cosh l)$. For the flank regions $E=K\left(2-\left(\gamma^{2}+2 \gamma \cos 2 h+\right.\right.$ $1)^{1 / 2}$ but this relation turns out not to be needed.

The above describes all possible types of barriers that can occur on any computation path. However the distribution of barriers according to their width and spacing between barriers can vary widely among different paths.

To apply this to a specific example, consider the initial state, shown in Figure 1 , with the head in state $|0\rangle$ in a wave packet localized to the left of the origin. All qubits are in state $|0\rangle$ except those at sites $0, N+1$ which are in state $|2\rangle$. The initial head and lattice qubit state are the path labels for this example. Iteration of $T$ on this state generates in turn all the integers as binary strings of length $\leq N$. When the space between the two markers is completely filled with $1 s$, corresponding to the integer $2^{N}-1$, the last pass of the head changes all $1 s$ to $0 s$. The head in state $|1\rangle$ then moves to the right away from the marker region as the enumeration is completed. A more detailed description of this process, based on iteration of $T$, is given elsewhere [13]. The potential 
Fig. 1. Initial and Final States for Counting GQTM for the First $2^{n}$ Binary Numbers. All lattice qubits are in state $|0\rangle$ except those at sites 0 and $n+1$ which are in state $|2\rangle$. The initial and final head states are shown as wave packets with internal head states $|0\rangle$ and $|1\rangle$ to the left and right respectively.

distribution associated with this example is obtained from a function $\mathcal{R}_{N}$ from the integers to $\{0,1\}$ such that $\mathcal{R}_{N}(m)=1$ if and only if the 5 th term of $T$, Eq. 10 is active in the transition $T|m, i\rangle \rightarrow|m+1, i\rangle$ (i.e a read-1 step). The potential distribution, obtained from $\mathcal{R}_{N}$ and Eq. 5 [14] is given by

$$
\left\langle m^{\prime}\left|V_{i}\right| m\right\rangle=K(1-\gamma)\left[\mathcal{R}_{N}(m) \delta_{m^{\prime}, m+1}+\mathcal{R}_{N}(m-1) \delta_{m^{\prime}, m-1}\right] .
$$

Note that for each component in the initial head wave packet state of Figure 1 there is a different function $\mathcal{R}_{N}$. This difference is ignored here because the functions differ only by a translation and describe the same potential distribution [13].

It turns out that the distributions of $1 s$ and $0 s$ in $\mathcal{R}_{N}$, exclusive of the 0 tails, can be represented as an initial segment of length $2^{N}$ of the heirarchical sequence $[16,17]$. If one defines the integers $\underline{m}$ as finite strings of $0 s$ and $1 s$ by $\underline{m}=01^{m} 0^{m+1}$ where $1^{m}$ and $0^{m+1}$ denote strings of $m 1 s$ and $m+10 s$, then (except for the 0 tails) $\mathcal{R}_{N}=\underline{0}, \underline{1}, \underline{0}, \underline{2}, \underline{0}, \underline{1}, \underline{0}, \underline{3}, \cdots, \underline{N}$. This definition of $\mathcal{R}_{N}$ is based on the initial state $|1, N+1, S\rangle$ with $|S\rangle$ as in Figure 1 chosen to be the path origin.

This sequence is an initial segment of length $2^{N}$ of the heirarchical sequence in the underlined numbers. Based on this it can be shown that $\mathcal{R}_{N}$ can be generated by a pair of recursion relations given by $[13,14]$

$$
\mathcal{R}_{n}=\mathcal{S}_{n-1} \underline{n} ; \mathcal{S}_{n}=\mathcal{R}_{n} \mathcal{S}_{n-1}
$$

for $n=1,2, \cdots, N$ with $\mathcal{S}_{0}=\underline{0}$.

Since the recursion relations generate the potential distribution it can be seen that the transfer matrix $Z_{N}$ is given by a pair of recursion relations obtained from Eq.12 as

$$
\begin{aligned}
& Z_{n}=W_{n} X_{n-1} \\
& X_{n}=X_{n-1} W_{n} X_{n-1}
\end{aligned}
$$


with $\left.X_{0}\right|_{11}=\mathrm{e}^{2 \mathrm{ik}}$ and $\left.X_{0}\right|_{12}=0$ for $n=1,2 \cdots, N . W_{n}$ is the transfer matrix for the potential corresponding to the sequence $\underline{n}=01^{n} 0^{n+1}$. Note that the order of matrix multiplication is the inverse of the order in which terms appear in the sequence of Eq. 12.

The main advantage of these recursion relations is that only polynomially many matrix multiplications are needed to obtain the elements of the matrix $Z_{N}$. If $Z_{N}$ is obtained from the matrices associated with each potential barrier in the distribution, then exponentially many matrix multiplications are required.

From Eq. 13 one sees that the only matrices needed in explicit form (other than $\left.X_{0}\right)$ are the $W_{m}$ for $m \leq N$. These are given explicitly by [14]

$$
\begin{aligned}
\left.W_{m}\right|_{11} & =\frac{\mathrm{e}^{\mathrm{ik}(\mathrm{m}+2)}}{2 \mathrm{i} \gamma \sin \mathrm{k} \sin \mathrm{l}}\left[\mathrm{e}^{2 \mathrm{ik}} \sin \mathrm{lm}-2 \gamma \mathrm{e}^{\mathrm{ik}} \sin \mathrm{l}(\mathrm{m}-1)+\gamma^{2} \sin \mathrm{l}(\mathrm{m}-2)\right] \\
\left.W_{m}\right|_{12} & =\frac{\mathrm{e}^{\mathrm{ikm}}}{2 \mathrm{i} \gamma \sin \mathrm{k} \sin \mathrm{l}}\left[\sin l m-2 \gamma \sin l(m-1) \cos k+\gamma^{2} \sin l(m-2)\right] .(14)
\end{aligned}
$$

Here the momenta $l, k$, which refer to the core and potential free regions, are related by $\cos k=\gamma \cos l$. Note that $\left.W_{m}\right|_{11}=\left.W_{m}^{*}\right|_{22},\left.W_{m}\right|_{21}=\left.W_{m}^{*}\right|_{12}$ and $W_{m}$ is unimodular.

These equations are valid for the unbound region $E \geq V$. The equivalent equations for the bound region are obtained by replacing sin everywhere in Eqs. 14 by i sinh, the hyperbolic sine function. Also $\cos k=\gamma \cosh l$.

\section{Landauer Resistance, Band Spectra}

Calculations are made here for the LR and band spectra for several different values of $\gamma$ and $N$ and momentum regions for the counting GQTM. The band spectra refer to the associated periodic system which is a crystal whose unit cell is the potential distribution corresponding to enumeration of the first $2^{N}$ integers. The momentum regions include the bound and unbound state regions. There are $\gamma$ dependent lower bounds on the values of $|k|$ below which there are no bands and the LR is very large [14]; these regions of $k$ values are excluded from the calculations.

Each figure gives a plot of the Log (base 10) of LR as a function of momentum $k$ for chosen values of $N$ and $\gamma$ and a region of $k$ values. The $\log L R$ is plotted instead of the LR because the LR fluctuates rapidly and violently over many orders of magnitude and the interest here is in regions of appreciable transmission (LR values of order unity). The band and gap spectra for the 
Fig. 2. The Log (base 10) of the Landauer Resistance Plotted as a Function of the Momentum for $N=10$ and $\gamma=0.999$ for a Momentum Range $0.0223 \leq k \leq 0.10$. The energy band spectrum for the associated periodic system is shown at the bottom of the figure where upper horizontal line segments correspond to energy bands and lower horizontal segments correspond to energy gaps. Very short bands or gaps appear as points. Band-gap edges show as vertical lines. The ordinal placement of the band spectrum, at the bottom of the figure, is done for convenience only.

same parameters is also included so that one can compare the LR fluctuations to the band spectra. The number of momentum values used to generate the curves in each figure ranges from about 3,000 to 8,000.

As both the LR and band spectra are extremely sensitive to values of $\gamma$, values that are reasonable physically should be chosen. This is done [14] by recalling that if $T$ is the bilateral shift on the qubit lattice, the Feynman Hamiltonian. Eq. 1 is the symmetrized lattice equivalent of $\left(-\hbar^{2} / 2 m\right) d^{2} / d x^{2}$. From this and $V=2 K \delta$ where $\delta=1-\gamma$, one obtains $\delta=V m \Delta^{2} / \hbar^{2}$ where $\Delta$ is the qubit lattice spacing. For electron systems, $m$ is of the order of the electron mass, $V$ is a few electron volts, and $\Delta$ is measured in Angstroms. Taking $m$ equal to 2 electron masses, $\Delta=1 \stackrel{\circ}{ }$, and $V=2 e v$ gives $\delta \simeq 0.001$ or $\gamma \simeq 0.999$.

Using this value of $\gamma$ the $\log L R$ and band spectra for the associated periodic system have been calculated for $N=10$ for several momentum or energy regions. The initial qubit state is shown in Figure 1. The results are shown in Figures 2 and 3 for momentum ranges of $0.0223 \leq k \leq 0.10$ and $0.095 \leq k \leq$ 0.220 . The bound state region extends up to $k=0.0447$ with the unbound region extending up and beyond the upper limit. In this and all succeeding figures the band spectrum is shown as a band with upper or lower flat line segments denoting bands or gaps respectively. Very narrow gaps (or bands) are shown as downward (or upward) pointing spikes. The results in the two figures show that the characteristics of the band spectra and $\log L R$ found for $k \leq 0.0447$ [14], extend smoothly into the region where $E>V$. Most of the region is occupied by bands with most gaps being quite narrow. A few wider gaps, such as those at $k=0.033,0.077,0.149$ and especially at $k=0.054,0.101,0.198$ are present.

The $\log L R$ can be characterized as a downward trending band of fluctuating values from an average value of about $-1,(L R=0.1)$ at the lower end of the $k$ region to about $-2,(L R=0.01)$ at the upper end of Figure 2 to a value of about -3.5 at the upper end of Figure 3. Several peaks extending to higher 
Fig. 3. The Log (base 10) of the Landauer Resistance Plotted as a Function of the Momentum for $N=10$ and $\gamma=0.999$ for a Momentum Range $0.095 \leq k \leq 0.220$. The energy band spectrum for the associated periodic system is shown at the bottom of the figure. Additional details are given in the caption for Figure 2.

Fig. 4. The Log (base 10) of the Landauer Resistance Plotted as a Function of the Momentum for $N=18$ and $\gamma=0.999$ for a Momentum Range $0.0223 \leq k \leq 0.10$. The energy band spectrum for the associated periodic system is shown at the bottom of the figure. Additional details are given in the caption for Figure 2.

values (up to $L R \simeq 10$ ) project out of the band.

The peaks in $\log L R$ appear to be associated with gaps, with the higher peaks associated with wider gaps. This effect [14] was also found for calculations with the Kronig- Penney model with potential distributions corresponding to the Fibonacci and Thue-Morse substitution sequences [20,21]. The minima in $\log L R$ which show as downward pointing spikes appear to be associated also with narrow gaps. Additional details on this are given elsewhere[14].

Results of calculations of the band spectrum and $\log L R$ for a larger value of $N=18$, corresponding to a potential distribution with $2^{17}$ individual potential barriers, are shown in the next two figures for the same two momentum ranges that were used in Figures 2 and 3. The same value of $\gamma=0.999$ was used. The band spectra are much more finely divided with each relatively broad band for $N=10$ divided into a great number of very narrow bands with intervening narrow gaps. The fraction of the momentum regions occupied by bands is smaller than that for $N=10$. The LR fluctuates very rapidly over many orders of magnitude between low values less than $10^{-4}$ to high values greater than $10^{20}$. For much of the momentum region $\log L R$ occupies a band (the black region in the figures) which trends down from a value around $0(L R=1)$ at the low momentum end of Figure 4 to a value around $-3\left(L R=10^{-3}\right)$ at the high momentum end of Figure 5. The trend, which represents a kind of average, is in the right direction as the LR should decrease as the energy is increased. 
Fig. 5. The Log (base 10) of the Landauer Resistance Plotted as a Function of the Momentum for $N=18$ and $\gamma=0.999$ for a Momentum Range $0.095 \leq k \leq 0.22$. The energy band spectrum for the associated periodic system is shown at the bottom of the figure. Additional details are given in the caption for Figure 2.

Fig. 6. The Log (base 10) of the Landauer Resistance Plotted as a Function of the Momentum for $N=10$ and $\gamma=0.99$ for a Momentum Range $0.069 \leq k \leq 0.22$. The energy band spectrum for the associated periodic system is shown at the bottom of the figure. Additional details are given in the caption for Figure 2.

Probably the most remarkable aspect is that the transmission probability is high $(>50-99 \%)$ on average, yet it is filled with numerous holes as small regions of very low or negligible transmission. This is shown by the numerous excursions of $\log L R$ to high values $>4$ from low average values around 0 to -2 and lower in Figures 4 and 5. It is noteworthy that this effect appears to be independent of whether $E \leq V$ or $E>V(E=V$ at $k=\arccos \gamma=0.0447)$. Also there is a correlation between the widths of regions of low transmission and the associated band spectrum gap widths in that wider gaps are correlated with wider regions of low transmission. Examples are the relatively wide band gaps at $k=0.054,0.100,0.198$. For these gaps the widths of the regions of very low transmission (high values of $\log L R$ ) are relatively wide.

Calculations made for the same values of $N=18$ and $\gamma=0.999$ for higher values of the momentum, up to $k=0.5$, show that the pattern described above continues. The "black" band containing much of the fluctuating values of $\log L R$ continues a slow downward trend to $\approx-4$ at the upper end of the region. Numerous narow spikes to high values are present. In some narrow momentum regions the spikes are so dense that high resolution calculations are needed for their individual resolution.

In order to investigate the dependence of the LR and band spectra on $\gamma$, calculations were made for a value of $\delta$ larger by a factor of 10 , i.e. $\gamma=0.99$, and $N=10$. The results for the energy regions $0.069 \leq k \leq 0.220$ and $0.20 \leq k \leq 0.42$ are shown in the next two figures. 
Fig. 7. The Log (base 10) of the Landauer Resistance Plotted as a Function of the Momentum for $N=10$ and $\gamma=0.99$ for a Momentum Range $0.20 \leq k \leq 0.42$. The energy band spectrum for the associated periodic system is shown at the bottom of the figure. Additional details are given in the caption for Figure 2.

The band spectra show the presence of a number of wide gaps. Also most of the bands are quite narrow. Comparison with the band spectra of Figs. 2 and 3, which are for the same value of $N$ but $\delta$ smaller by a factor of 10 , show that the fraction of the momentum regions occupied by bands is much lower and gap widths are wider. $\log L R$ shows a band of fluctuating values decreasing slowly from an average value of $\approx 1(L R=10)$ at the low end of the momentum region in Figure 6 to a value of $\approx-2(L R=0.01)$ at the upper end of the momentum region in Figure 7.

Superimposed on this are peaks in the values of $\log L R$ which extend up to 18 or more. Each peak appears to occupy the same momentum region as the gap, with the peak height correlated with the gap width in that wider gaps are associated with higher peaks. This is especially clear in Figure 6. The distribution of peak heights in the figure also shows a heirarchical relationship similar to that shown by the potential distribution of Eqs. 11 and 12. That is, the region between the two largest peaks is divided in two by a peak of lesser height. Each subregion is divided in two by an even smaller peak. This division into two subregions by peaks of decreasing height continues down to the smallest peaks associated with the small gaps and which form the band of fluctuating values. This regularity is less evident in the momentum region shown in Figure 7 for which the fluctuations in $\log L R$ are more chaotic. Since the value of $k=0.142$ for which $E=V$ occurs in the middle of Figure 6 , it appears that the regularity of the peak distribution is not dependent on whether the momentum is in the bound or unbound region.

In order to investigate in more detail the relation between peaks in $\log L R$ and bands and gaps, a high resolution calculation for $\gamma=0.99, N=10$ was done for the small momentum region $0.21 \leq k \leq 0.23$. The results are shown in the next figure. The results show that each peak in $\log L R$ is defined by sharp minima which are associated with relatively narrow gaps. More exactly the minima seem to be associated with the band-above-gap edges of the narrower gaps. In this fashion the momentum region of each peak includes one band and one gap. Each gap has a sharp minima associated with its upper edge except for the wider gaps. This appears to result from the fact that in general the 
Fig. 8. The Log (base 10) of the Landauer Resistance Plotted as a Function of the Momentum for $N=10$ and $\gamma=0.99$ for a Small Momentum Range $0.21 \leq k \leq 0.23$. The energy band spectrum for the associated periodic system is shown at the bottom of the figure. Additional details are given in the caption for Figure 2.

Fig. 9. The Log (base 10) of the Landauer Resistance Plotted as a Function of the Momentum for $N=8$ and $\gamma=0.9$ for a Momentum Range $0.18 \leq k \leq 0.52$. The energy band spectrum for the associated periodic system is shown at the bottom of the figure. Additional details are given in the caption for Figure 2.

Fig. 10. The Log (base 10) of the Landauer Resistance Plotted as a Function of the Momentum for $N=8$ and $\gamma=0.9$ for a Momentum Range $0.5 \leq k \leq 1.0$. The energy band spectrum for the associated periodic system is shown at the bottom of the figure. Additional details are given in the caption for Figure 2.

depth of the minima in $\log L R$ decreases with increasing width of the gap at whose edge it is located until it disappears entirely for sufficiently wide gaps. For these wider gaps the momentum region of the associated peak includes two bands and two gaps. This effect, which was noted before [14], is seen for the wide gaps centered at $k=0.216,0.2185,0.221,0.2267$.

Increasing $\delta$ by another factor of 10 to 0.1 with $\gamma=0.9$ gives the results shown in the next two figures for the momentum regions $0.18 \leq k \leq 0.52$ and $0.5 \leq k \leq 1.0$ respectively. The calculations are for $N=8$. Since $k=0.45$ for $E=V$, the energy region used in Figure 9 is mostly the bound state region. That used in Figure 10 includes part of the unbound state region. The results in the figures show that the potential is high enough (10 times the height of that in Figures 6 and 7 ) so that definite differences in the band spectra 
and $\log L R$ are evident for the two regions. In the bound state region (Fig. 9 ) the bands are extremely narrow and are few and widely separated with many large gaps present. The values of $\log L R$ show the the $L R$ is very high for most of the region, except for a few very narrow spike minima to values around $1,(\log L R=0)$. The spike minima occur at the same energies as the bands.

In the unbound region (Fig. 10) the bands are much more numerous and individual bands are wider than in the bound state region. The LR is much lower on average, and there are extended regions $(0.59 \leq k \leq 0.62$ and $0.70 \leq k \leq 0.81$ ) for which $L R \simeq 1$. To summarize, the results for these figures show that in the bound state region there is almost no transmission through the 128 potentials except for a few extremely narrow regions at which bands are located. In the unbound state region there are wide regions of good transmission of $\simeq 50 \%$ or more. Energy bands are more numerous and are wider.

\section{6 discussion}

The values of $\gamma$ and $N$ for which results of calculations of band spectra and $\log L R$ have been shown are quite limited. These limitations are imposed by the requirement that the calcuations of the band spectra and LR be reasonably reliable. This is not trivial because fluctuations in the values of the matrix elements of $Z_{N}$ used to calcuate the LR and the band spectra become extremely rapid and extreme at higher values of $N$ and lower values of $\gamma$. For example, even for $N=18$ and $\gamma=0.999$ or $N=8$ and $\gamma=0.9, \operatorname{Tr} Z_{N}$ and the LR fluctuate rapidly between $\pm 10^{M}$ with $M$ taking values of $200-300$ or more for some values of $k$. Also these fluctuations occur over extremely small intervals of $k$.

In essence limitations on the values of $N$ and $\gamma$ are imposed by (classical) computer program limitations on the magnitude and number of significant figures allowed in the computations. Modifications of the programs to allow calculations over a larger range of $N$ and $\gamma$ values are not warranted as they are complex and at most give one a slight extension of the range of acceptable values of $N$ and $\gamma$.

In many ways the results obtained here, which are similar to those obtained by Roy and Khan [20,21], are unexpected. The LR fluctuates rapidly with many spikes down to extremely low values over the momentum range examined. Except for the highest potential considered (i.e. $\gamma=0.9$ ) there is not much difference between the bound and unbound regions as far as the band spectra or $\log L R$ are concerned. The transmission, even in the bound region for $N=$ 
18 and $\gamma=0.999$, is high $(\simeq 50 \%$ or more $)$ on average although there are a great many holes of extremely low transmission.

These results need explaining since one would expect little or no transmission in the bound region, because of decay in the transmission amplitude resulting from tunnelling effects and localization [10]. This is especially the case for $N=18$ with more than 128,000 potential barriers present. Also one would expect almost complete transmission in the unbound regions with few or no regions of low transmission. Neither of these results occur.

It is suggested here that the calculated results are best understood in terms of coherent superposition of the many reflected waves moving backwards through the potential distribution. At some values of the momenta the reflected waves interfere constructively, which greatly increases the amplitude of the overall reflected component and decreases the amplitude to the transmitted component. At other momentum values the reflected waves interfere destructively. In this case the amplitude of the reflected component is small and the amplitude of the transmitted component is high. $\square$ Note that if $B$ and $F$ are the complex valued reflection and transmission coefficients respectively for the potential distribution,Eqs. 6 and 7, then normalization requires that $|B|^{2}+|F|^{2}=1$.

The number of component waves which add coherently should be very large. Since each barrier in the distribution generates transmitted and reflected components from any impinging wave, whether it is the input wave, or waves reflected or transmitted from other barriers, the number of multiple reflections or transmissions or combinations of the two becomes extremely large, especially in a distribution with many barriers. All the components moving in the same direction add coherently.

This coherent superposition is expected to depend sensitively on all parameters involved. It also should be very dependent on the fact that the potential distribution is not random; in the example considered here the distribution is quasiperiodic $[24,25]$ and is heirarchical. Intuitively if coherent superposition is the dominant effect, one would expect that for fixed potential height (fixed $\gamma$ ), the fluctuation rate of the Landauer Resistance and the number of bands and gaps to increase with increasing $N$. The reason is that the number of individual potential barriers present increases exponentially with increasing $N$. The corresponding increase in the number of reflected and transmitted component waves which combine coherently means that momentum regions in which backward moving reflections interfere destructively to give high transmission

1 Exactly the same argument applies mutatis mutandis. Constructive or destructive interference of the transmitted components decreases or increases the amplitude of the reflected component emerging from the distribution. From the viewpoint of a computation one is more interested in the amplitude of the transmitted component than in the reflected one. 
Fig. 11. The magnitudes of the Reflection (B) and Transmission (F) Coefficients for a Single Potential Barrier Corresponding to $m=10$ read 1 Steps for $\gamma=0.999$ for a Momentum Range of $0 \leq k \leq 0.5$. The values of $|B|$ for $k \geq 0.3$ were increased by a factor of 10 to show more clearly the nodal structure.

become much narrower and broad bands split into many narrower bands with intervening gaps.

In a similar fashion momentum regions in which backward moving reflections combine constructively to give very low transmission also become narrower. This narrowing and splitting of regions with constructive or destructive interference results in a large increase in the extent and rapidity of fluctuations in the LR and splitting of the energy bands.

The importance of coherent effects in the transmission and reflection of a state through the potential distribution is demonstrated clearly by consideration of the transmission and reflection for a single potential barrier. For motion in the potential distribution corresponding to iteration of all binary numbers of length $\leq N$ the complex transmission $(\mathrm{F})$ and reflection $(\mathrm{B})$ coefficients are obtained from Eqs. 7 and 8 and the normalization $|F|^{2}+|B|^{2}=1$ as

$$
F=\frac{1}{\left.Z_{N}\right|_{22}}, B=-\frac{\left.Z_{N}\right|_{12}}{\left.Z_{N}\right|_{22}}
$$

For a single barrier corresponding to $m$ read 1 steps these equations hold with $Z_{N}$ replaced by $W_{m}$, Eq. 14 . The momentum dependence of the magnitudes and phases of $\mathrm{F}$ and $\mathrm{B}$ for $m=10$ and $\gamma=0.999$ for a momentum range of $0 \leq k \leq 0.5$ are shown in the next two figures. The magnitude values for the reflection coefficient $B$ for $k \geq 0.3$ have been multiplied by a factor of 10 to illustrate the nodal structure of $B$ more clearly. The range of the phases in Figure 12 extends from $-\pi$ to $\pi$. The large discontinuities in the curves for both $\mathrm{B}$ and $\mathrm{F}$ are a artifact of the presentation method in that $\pi+x \equiv-\pi+x$. The discontinuity in the phase for $\mathrm{B}$ at $k \simeq 0.3$ is real, though. It occurs at the node point for $|B|$, Figure 11. The figures show that the magnitudes of $\mathrm{B}$ and $\mathrm{F}$ tend rapidly to values close to 0 and 1 . At a value of $k=0.0447$ corresponding to $E+V,|F|=0.976$ and $|B|=0.218$. Thus at momenta corresponding to energies above the barrier height there is still appreciable reflection. Unlike the magnitudes the phases of both $\mathrm{B}$ and $\mathrm{F}$ vary steadily over the range of momenta shown. This is significant because it means that 
Fig. 12. The Phases of the Reflection (B) and Transmission (F) Coefficients for a Single Potential Barrier Corresponding to $m=10$ read 1 Steps for $\gamma=0.999$ for a Phase Range of $[-\pi, \pi]$. The large discontinuities in the phases for $B$ and $F$ are a result of the figure presentation in which $-\pi$ and $\pi$ are not identified.

coherent additions of the many reflected and transmitted components in a sequence of these barriers would be expected to give rapidly varying overall transmission and reflection coefficients. This is shown by the calculations.

Calculations show that as $N$ is increased for constant $\gamma$ the internodal maxima increase and the distances between nodes in $|B|$ decrease. The rate of change of the phases of both $\mathrm{F}$ and $\mathrm{B}$ also increases and the discontinuities at the $\mathrm{B}$ node points increase. This implies an increase in the rapidity of fluctuations of the LR and in the number of spectral bands and gaps with increasing $N$, also in agreement with the calculations.

As the potential is increased ( $\gamma$ decreased) but $N$ is fixed, the position of the first node in $|B|$ moves out to higher values of $k$. Also the internodal maxima increase. However the rates of change of the phases for both $\mathrm{B}$ and $\mathrm{F}$ decrease out to the first node as the potential height increases. For higher values of $k$ the phase slopes appear to be roughly independent of the potential height. It is of interest to note that the position of the first node at $k=0.59$ for $\gamma=0.9, N=8$ is close to the value of $k$ at which the LR and band spectra change character (See discussion of Figures 9 and 10).

The curves of Figure 11 can be used to show the importance of coherent effects. Consider for example the potential distribution for $N=18$ and $\gamma=0.999$ used to calculate the LR and band spectra shown in Figures 4 and 5. The properties of the heirarchical distribution are such that for $m=1,2, \cdots, N-1$ there are $2^{N-m-1}$ potential barriers of width corresponding to $m$ read 1 steps in the distribution. For the example at hand there are 128 barriers for $m=10$. If the reflections and transmissions from these barriers combine incoherently, then the probability of transmission through just these barriers and ignoring the others in the distribution is given by $\left(1-|B(k)|^{2}\right)^{128}$ where the $k$ dependence is shown explicitly. It is clear from the $k$ dependence of $|B(k)|$ shown in Figure 12 and the LR shown in Figures 4 and 5 that the $k$ dependence of the probability of transmission is completely different from the incoherent prediction. Inclusion of the effects of the other barriers for different $m$ in the incoherent prediction whould make the disagreement even more extreme. 
The coherent effects implied in the results for the LR and band spectra give a good illustration of nonlocality in quantum mechanics. Consider a value of $k$ at which the reflection components interfere destructively as they move backwards along the path. This means the amplitude of the overall reflection coefficient for the reflected wave emerging from the distribution is a (local) minimum and the amplitude for the overall emerging transmitted wave is a maximum. This is a nonlocal effect since much of what happens to the reflected components occurs at path locations distant from those of the transmitted components. The effect is quite pronounced since it extends over the whole potential distribution. In the case of $N=18$ this effect extends over $10^{6}$ path sites. The distance over which this effect occurs grows exponentially with $N$ as the number of path sites in the potential distribution is about equal to $2^{N+2}$.

Is is to be emphasized that this effect is quite general and is not restricted to the specific distribution considered here. It is shown, for instance, in the graphs of the LR versus energy for different potential distributions obtained by Roy and Kahn [20,21]. These effects also play an important role in most work on transmission of electrons through nonrandom potential distributions.

\section{Acknowledgements}

This work is supported by the U.S. Department of Energy, Nuclear Physics Division, under contract W-31-109-ENG-38. 


\section{References}

[1] P. Shor, in Proceedings of the 35th Annual Symposium on the Foundations of Computer Science (IEEE Computer Society, Los Alamitos, CA 1994), p. 124. For a recent review see A. Ekert and R. Jozsa, Rev. Mod. Phys. 68733 (1996).

[2] M. Boyer, G. Brassard, P. Høyer, and A. Tapp, Tight Bounds on Quantum Searching, Los Alamos Archives Preprint quant-ph/9605034; L.K. Grover, A fast quantum mechanical algorithm for database search, Proceedings of the 28th annual ACM Symposium on Theory of Computing, Philadelphia, PA, 1996, pp $212-219$.

[3] D. Deutsch and R. Josza, Proc. Roy. Soc. (Lond.) A439, 553 (1992).

[4] S. Lloyd, Science 2731073 (1996); C. Zalka Efficient Simulation of Quantum Sysytems by Quantum Computers, Report No. BUTP-96/11, March 25, 1996, Los Alamos Archives quant-ph/9603026.

[5] R. Feynman, Int Jour. Theoret. Phys. 21467 (1982).

[6] P. W. Shor, Phys. Rev. A 52 R2493 (1990); R. LaFlamme, C. Miquel, J.P. Paz, and W. H. Zurek, Phys. Rev. Letters 77198 (1996).

[7] S. Lloyd, Phys. Rev. Letters, bf 75346 (1995); D. P. DiVencenzo, Phys. Rev. A 511015 (1995); D. Deutsch, A. Barenco, and A. Ekert, Proc. Roy. Soc. London A 449669 (1995).

[8] D. Deutsch, Proc. Roy. Soc. London Ser. A 40097 (1985); 42573 (1989).

[9] P. Benioff, Jour. Stat. Phys. 22563 (1980); Ann. NY Acad. Sci. 480475 (1986).

[10] R. Landauer, Philos. Trans. Roy. Soc. London A353 367 91995); Physics Letters A 217188 (1996); Physics Today 44 No. 5, 23 (1991).

[11] A. Peres, Phys. Rev A 323266 (1985)

[12] W. H. Zurek, Phys. Rev. Lett. 53391 (1984)

[13] P. Benioff, Phys. Rev. Letters, to Appear

[14] P. Benioff, Submitted to Phys. Rev. B.

[15] R. P. Feynman, Optics News 1111 (1985); reprinted in Foundations of Physics 16507 (1986).

[16] B. A. Huberman and M. Kerzberg, J. Phys. A: Math. Gen. 18 L331 (1985)

[17] F. Igl oi and L. Turban, Los Alamos Archives cond- mat/9606118).

[18] R. Landauer, Phil. Mag. 21 863, (1970).

[19] P. Erdos and R. C. Herndon, Adv. Phys, 31 65, (1982)

[20] C. Roy and A. Khan, Phys. Rev. B, 4914979 (1994): 
[21] C. Roy and A. Khan, Solid State Commun. 92241 (1994).

[22] P. Benioff, Phys. Rev A 541106 (1996).

[23] For example: M. Kohmoto, L. P. Kadanoff, and C. Tang, Phys. Rev. Letters 50 1870 (1983); M. Kohmoto, International Jour. Modern Phys. 131 (1987); G. Gumbs and M. K. Ali, Phys. Rev. Letters 601081 (1988); M. Kolar and M. K. Ali, Phys. Rev B 39426 (1989).

[24] T. Fujiwara and T. Ogawa (Eds) Quasicrystals, Proceedings of the 112th Taniguchi Symposium, Shima, Mie Prefecture, Japan Nov 14-19 1989, Springer Series in Solid State Sciences 93, Springer Verlag New York.

[25] D. DiVincenzo and P. Steinhardt Eds., Quasicrystals the State of the Art, in Directions in Condensed Matter Physics-Vol. 11, World Scientific Singapore 1991. 


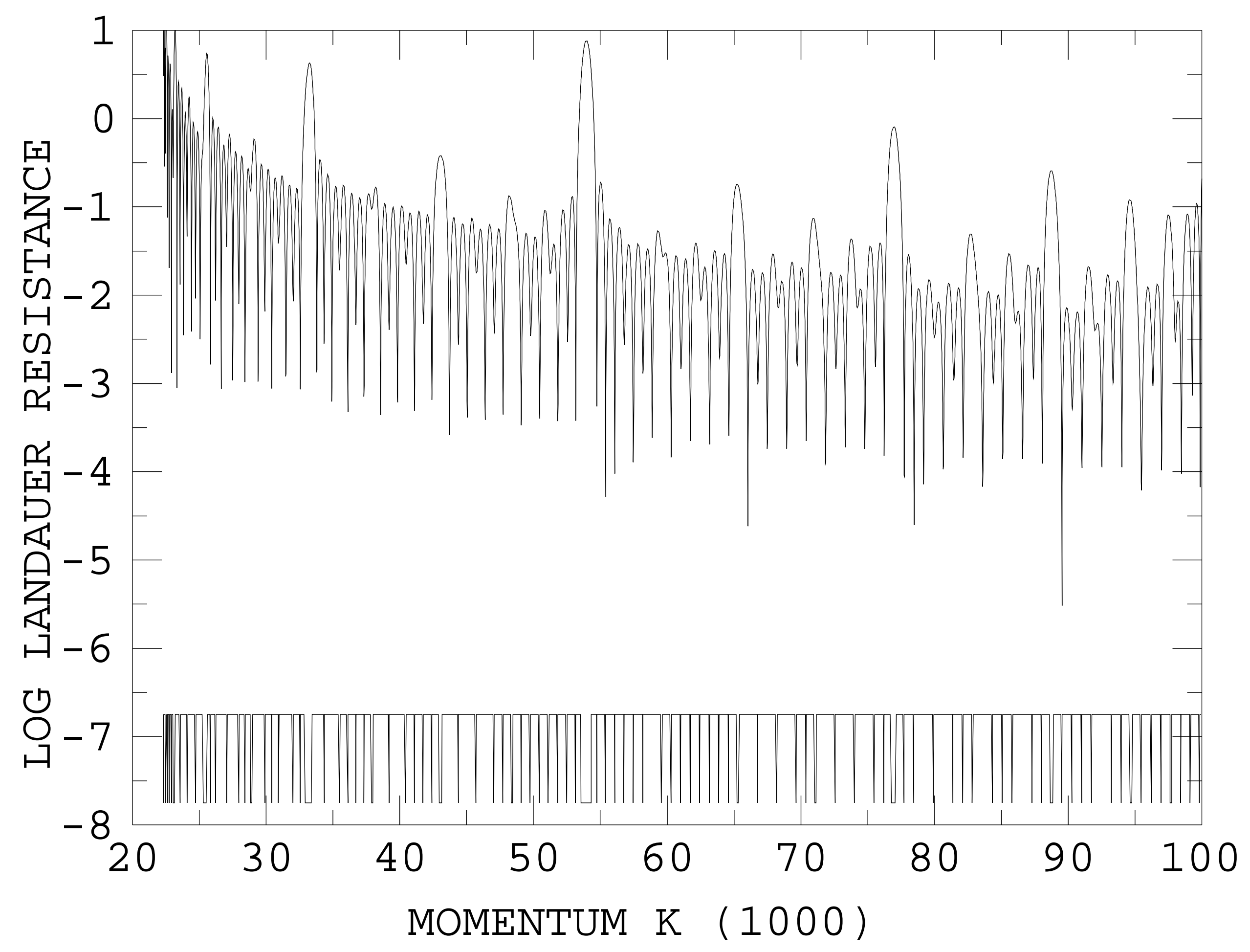




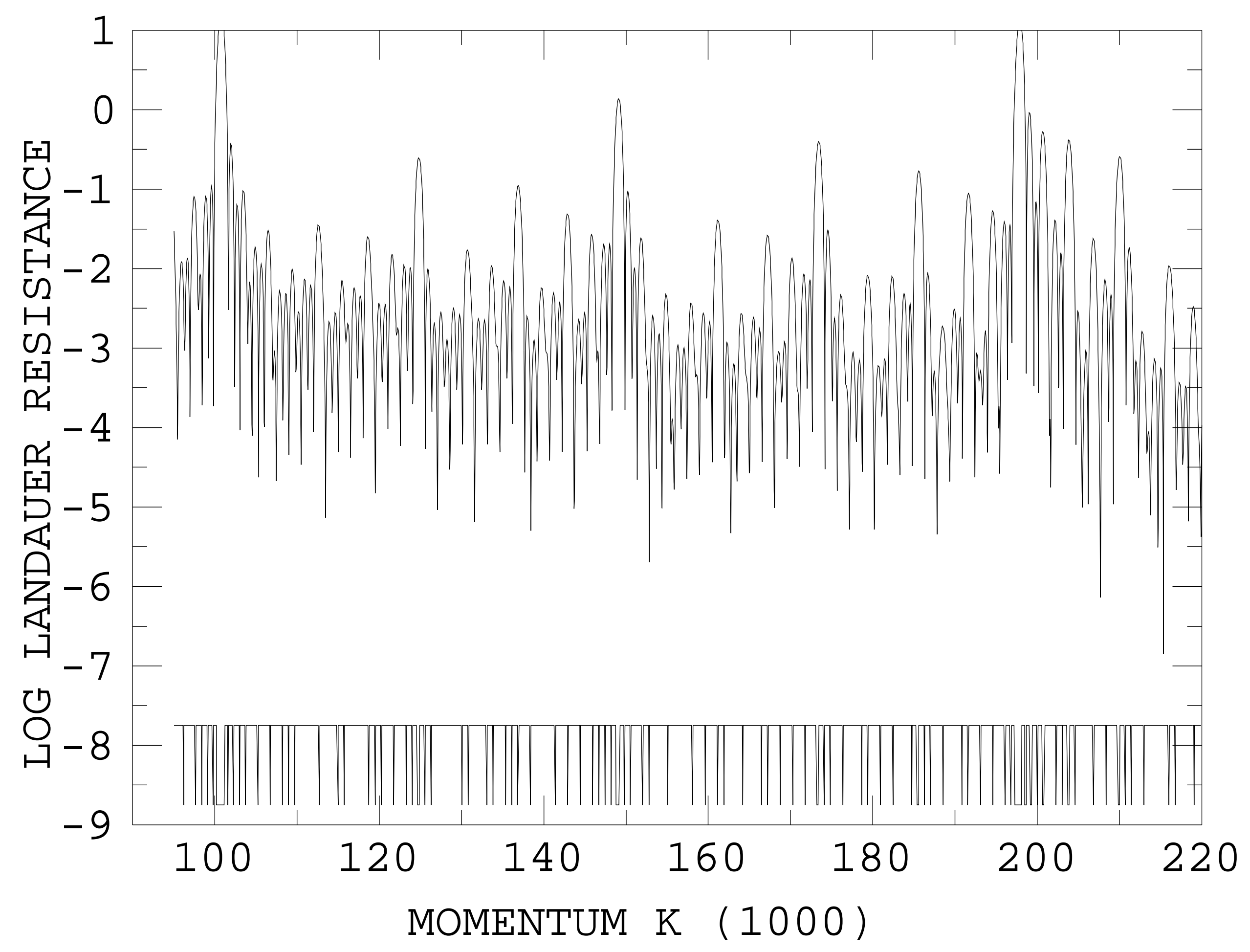




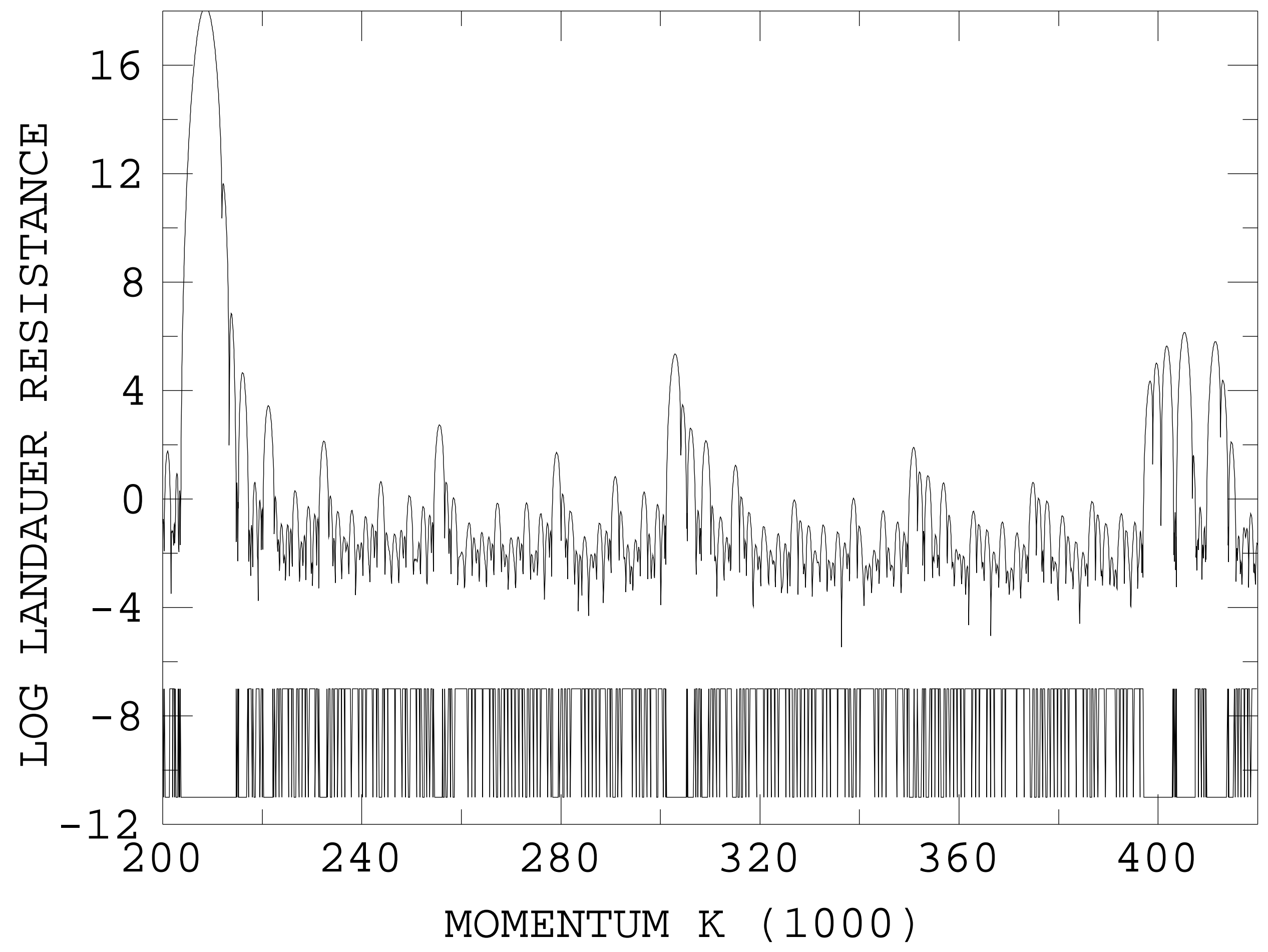




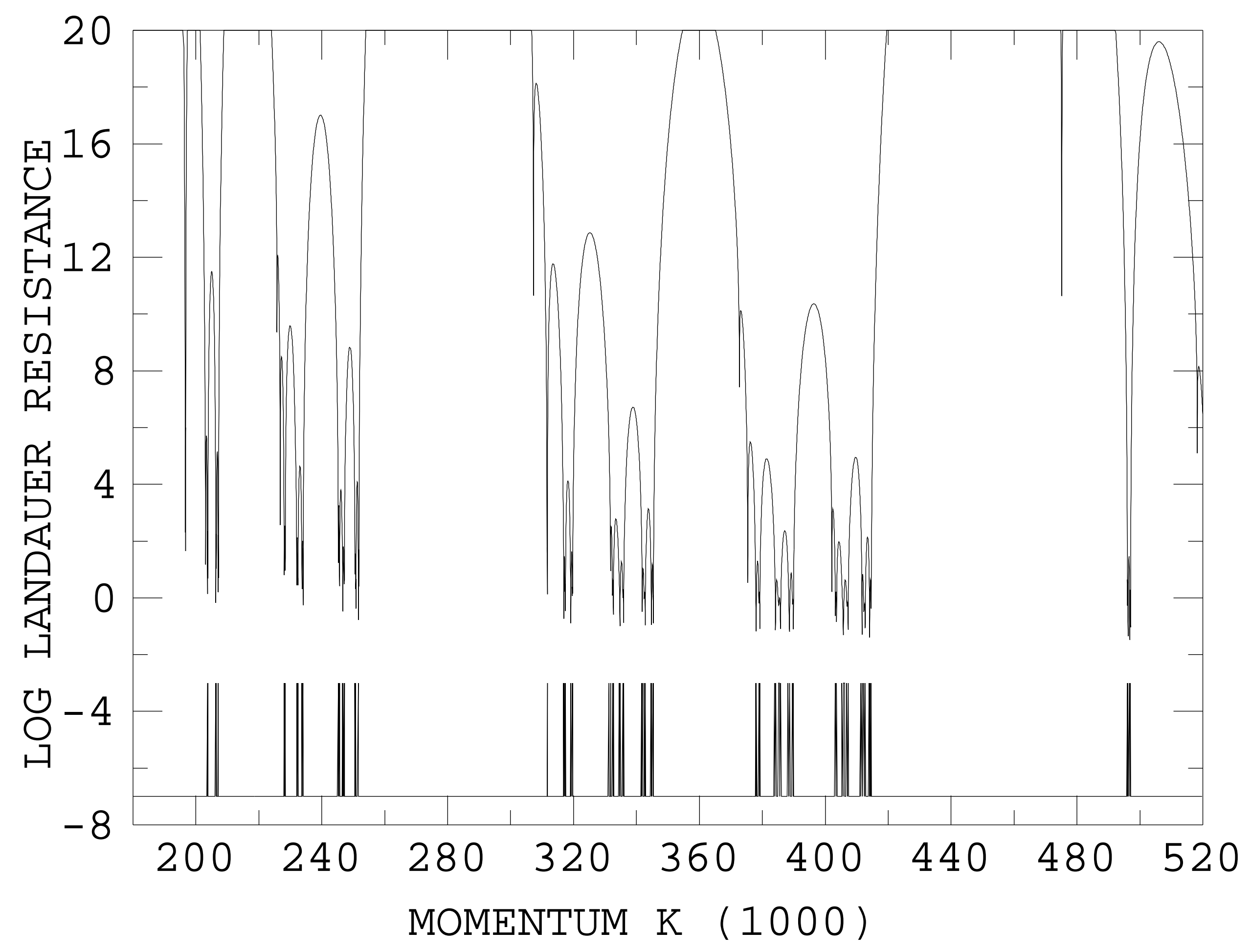




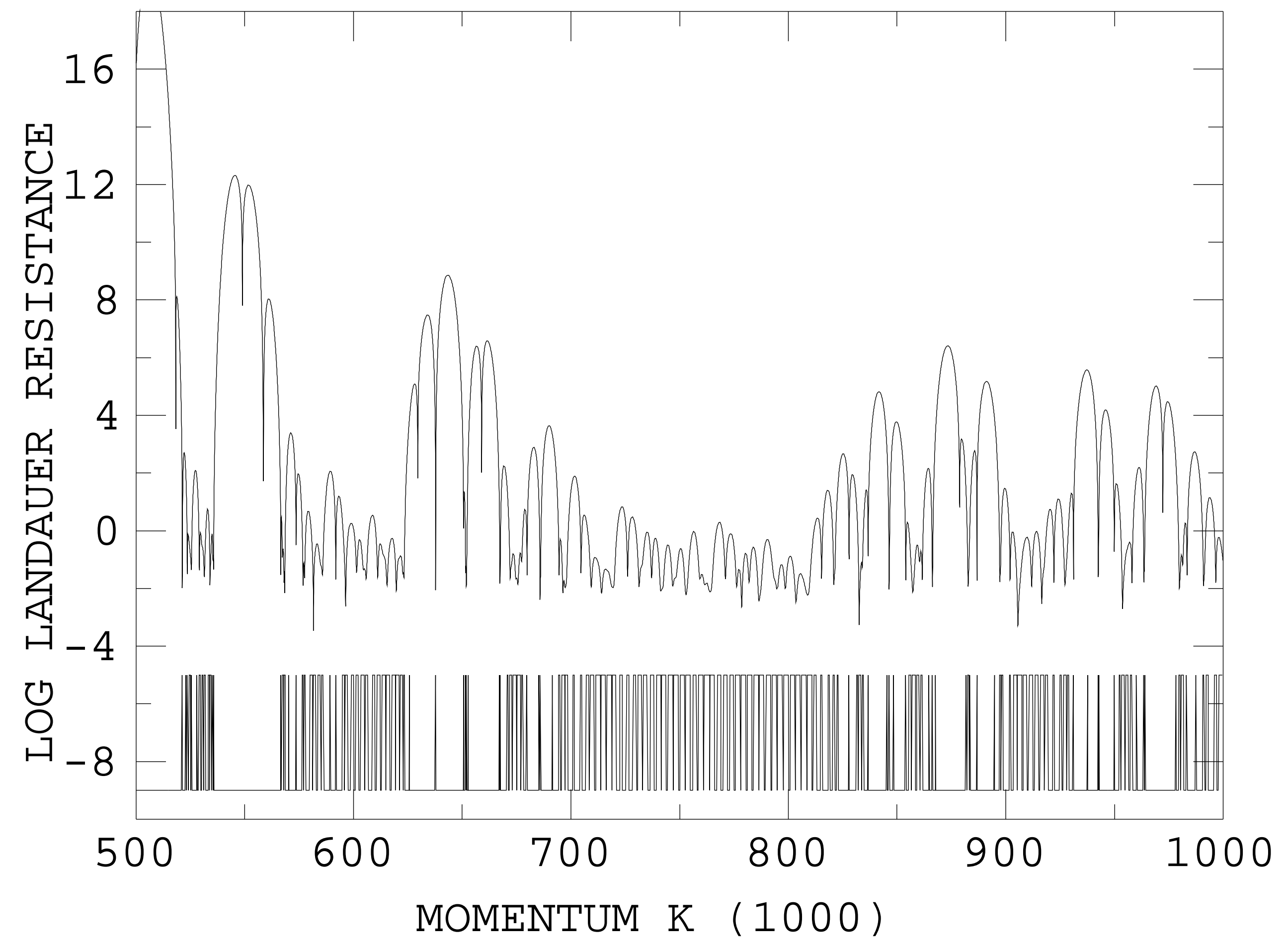




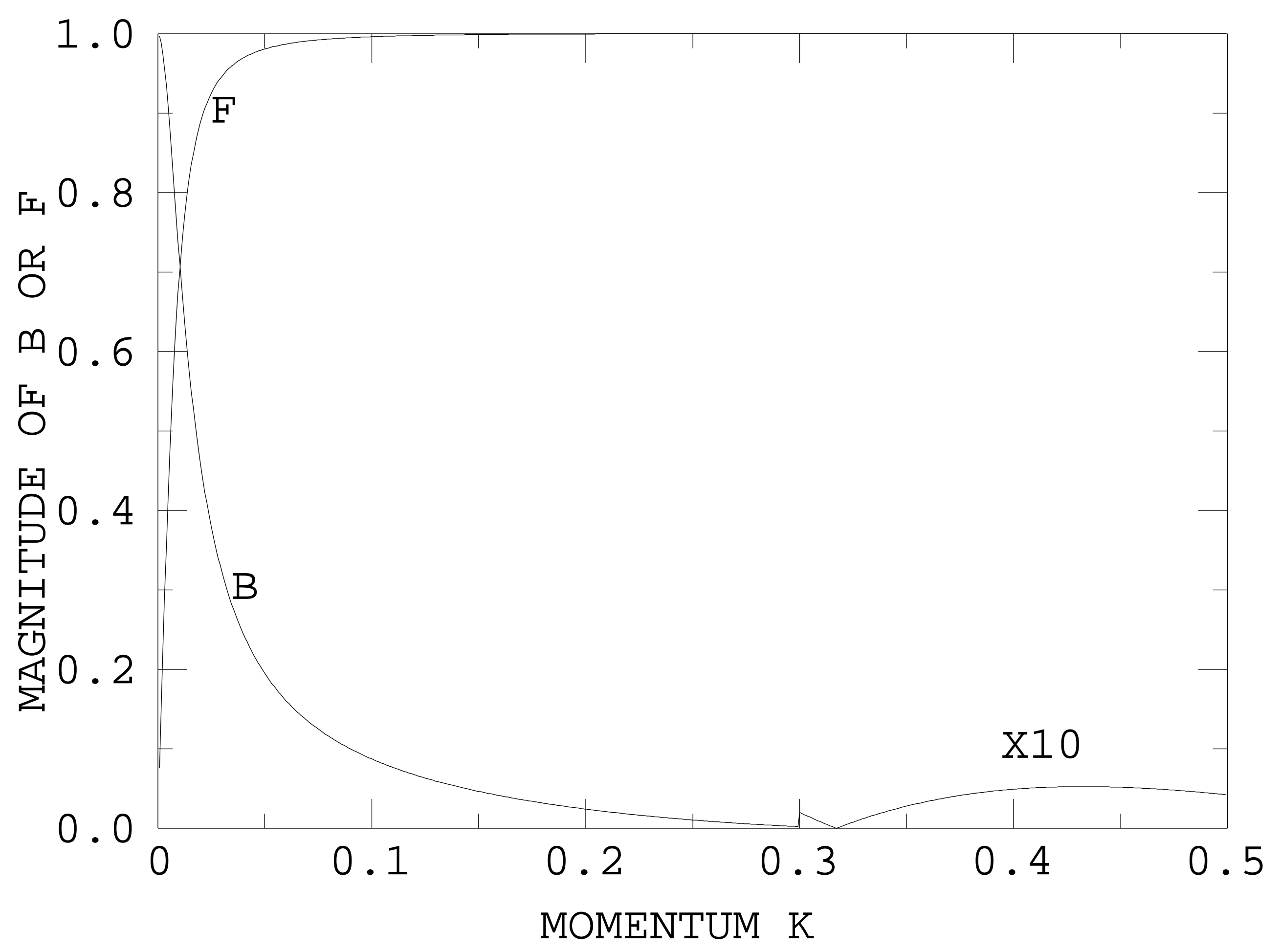




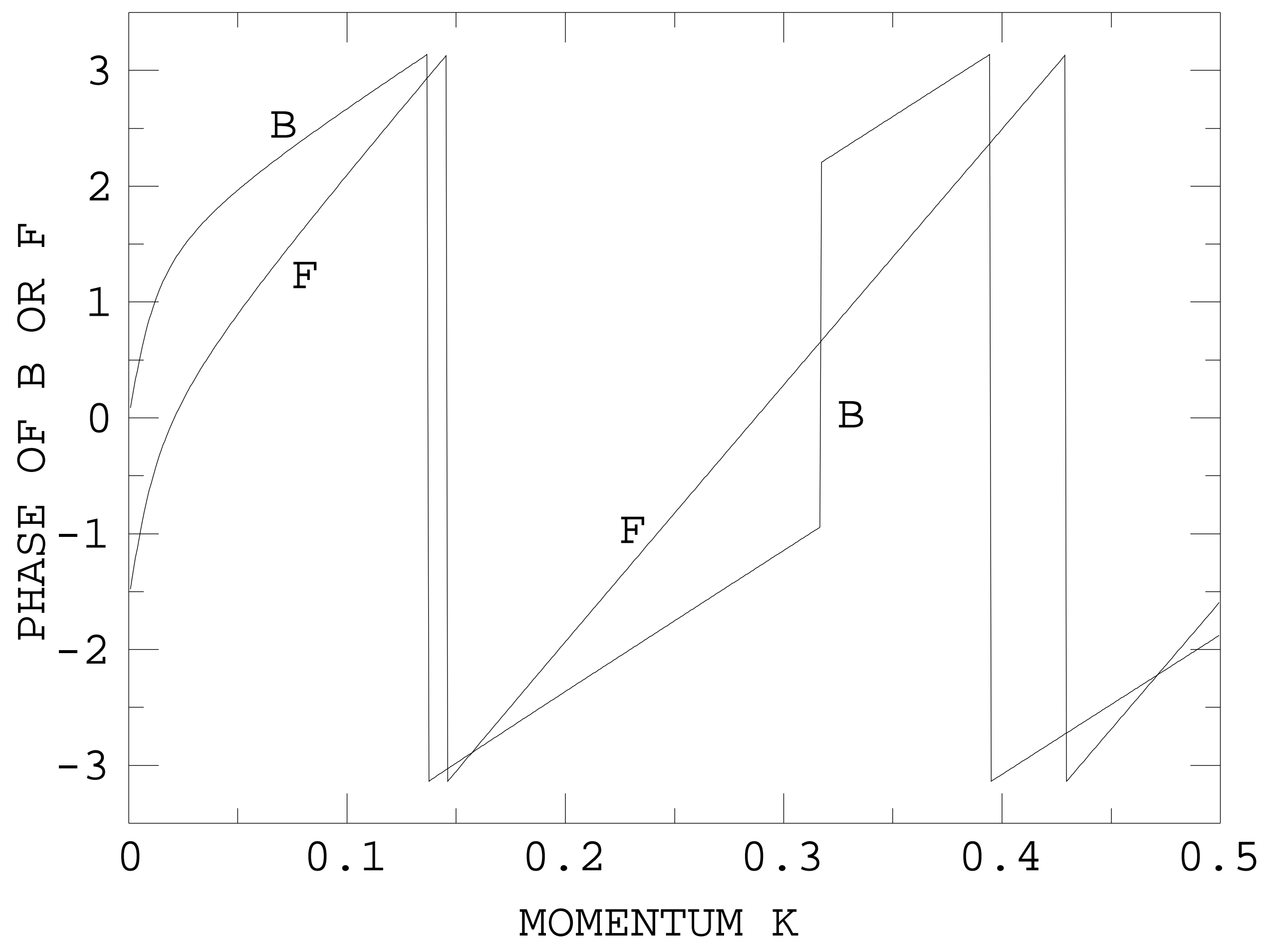

